\title{
Chemical colitis caused by peracetic acid or hydrogen peroxide: a challenging dilemma
}

Peroxide agents used to disinfect endoscopes may cause chemical colitis [1-3]. However, it remains controversial whether the agent reponsible is the peracetic acid or the hydrogen peroxide in the solution. We recently encountered four cases of chemical colitis, showing characteristic morphologic and histologic features, including the "snow white sign", following use of a new automated disinfection device in our endoscopy unit. The disinfectant contained $0.2 \%$ peracetic acid as the active substance and $3 \%$ hydrogen peroxide as the auxiliary substance. All the cases occurred during a 4-day period, and all the patients showed mucosal changes in the various segments of the colon (see - Table 1) after washing with water to remove any fecal material. The lesions manifesting as the "snow white sign" consisted of raised whitish-yellow plaques of varying sizes, some of which were confluent ( $\bullet$ Fig. $1, \bullet$ Video 1 ). Vigorous effervescence was also noted. All biopsy specimens from the plaques showed the same histological pattern, compatible with

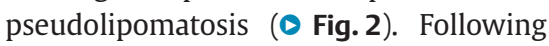
colonoscopy no specific gastrointestinal symptoms were observed in any of the patients, and they all remained asymptomatic at a review visit 3 weeks later. Because the lesions were expected to heal spontaneously $[1,2]$, follow-up colonoscopy was not carried out. A programming error was discovered in the automated disinfection device, related to the rinsing of the air/water channels of the endoscopes. After correction of the problem there have been no more cases of chemical colitis in our department.

\section{Video 1}

Endoscopic view of the "snow white sign" (case 3).

\begin{tabular}{lllll}
\hline \multicolumn{2}{l}{ Table 1} & Patient characteristics. & & \\
$\begin{array}{l}\text { Patient } \\
\text { no. }\end{array}$ & Sex & $\begin{array}{l}\text { Age } \\
\text { (years) }\end{array}$ & Indication for colonoscopy & Location of lesions \\
1 & M & 73 & Surveillance for colon carcinoma & Hepatic flexure \\
\hline 2 & M & 56 & Constipation & Splenic flexure \\
\hline 3 & F & 70 & Abdominal pain & Hepatic flexure \\
\hline 4 & M & 61 & Constipation and hematochezia & Ascending colon \\
\hline
\end{tabular}

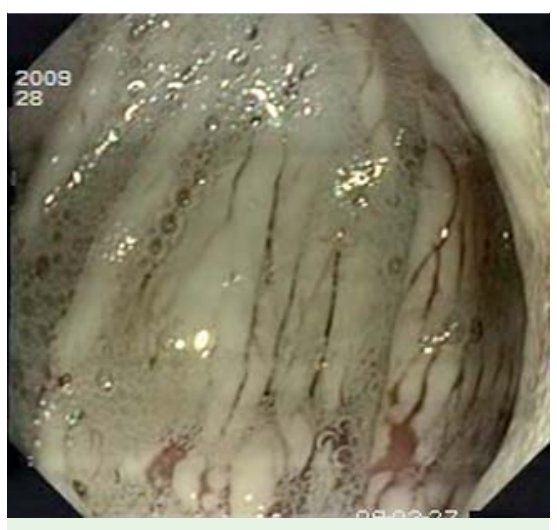

Fig. 1 Typical endoscopic appearance of the "snow white sign" (case 3).

Peracetic acid consists of an aqueous solution of acetic acid and hydrogen peroxide. When peracetic acid dissolves in water, it disintegrates into hydrogen peroxide and acetic acid (peracetic acid + water $\longleftrightarrow$ hydrogen peroxide + acetic acid), which, in turn, decompose into water, oxygen, and carbon dioxide. Thus we could expect that some of the formed hydrogen peroxide may induce chemical colitis, even when it is only present as part of a peracetic acid-based disinfectant. Indeed, like in our cases, Morini et al. [1] also noted the snow white sign in the area of the mucosa that was washed with water. These observations point to the possibility that the hydrogen peroxide formed when peracetic acid dissolves in water causes the relat-

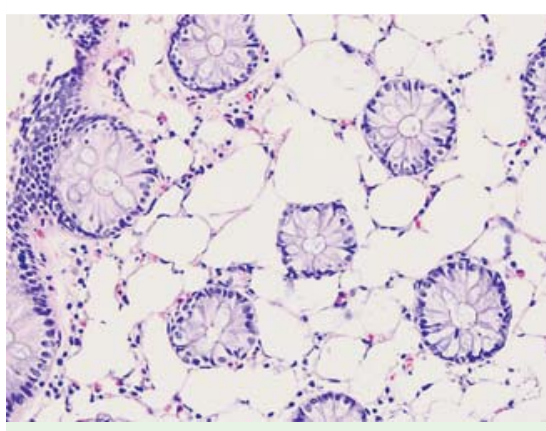

Fig. 2 Histologic appearance of colonic pseudolipomatosis, showing empty vacuoles within the submucosa (case 1) (hematoxylin and eosin, original magnification $\times 400$ ).

ed colitis. Furthermore, in another report, Coton et al. [4] described the development of acute colitis following the use of a disinfectant containing peracetic acid (Anioxyde $\left.1000^{\circledR}\right)$, in which hydrogen peroxide was used as an activator. In that case, the observed mucosal lesions showing the characteristic snow white pattern could be related to the residual hydrogen peroxide content in the activated solution. Clearly, this unique form of colitis seems more likely to be due to hydrogen peroxide. Experimental studies are required, similar to those carried out with hydrogen peroxide [5], to evaluate reproduction of the lesions with peracetic acid.

Endoscopy_UCTN_Code_CPL_1AJ_2AB Endoscopy_UCTN_Code_CCL_1AD_2AJ 


\section{Kara, I. Turan, Z. Polat, T. Dogru,}

\section{S. Bagci}

Gulhane Medical School, Division of

Gastroenterology, Ankara, Turkey

\section{References}

1 Morini S, Campo SMA, Zullo A et al. Chemical colitis induced by peracetic acid: further evidence. Endoscopy 2009; 41: 383

2 Cammarota G, Cesaro P, Cazzato A et al. Hydrogen peroxide-related colitis (previously known as "pseudolipomatosis"): a series of cases occurring in an epidemic pattern. Endoscopy 2007; 39: 916-919
3 Lapeyre $B$. The "frost sign" and the "snow white sign": intramucosal air injection or peroxide colitis? Endoscopy 2005; 37: 679

4 Coton T, Bohand X, Guisset M et al. Acute colitis induced by a peracetic acid-based solution used to disinfect endoscopes. Gastroenterol Clin Biol 2003; 27: 556-558

5 Sheehan JF, Brunjolfsson G. Ulcerative colitis following hydrogen peroxide enema: a case report and experimental production with transient emphysema of colonic wall and gas embolism. Lab Invest 1960; 9: 151-168

\section{Bibliography}

DOI $10.1055 / \mathrm{s}-0029-1215260$

Endoscopy 2010; 42: E3 - E4

(c) Georg Thieme Verlag KG Stuttgart · New York . ISSN 0013-726X

\section{Corresponding author}

\section{Kara, MD}

GATA Gastroenteroloji Bilim Dali Etlik 06010

Ankara

Turkey

Fax: +90-312-3042010

drmuammerkara70@hotmail.com 